\title{
Characteristics of Antioxidative Flavonoid Glycosides in Lemon Fruit
}

\author{
Yoshiaki MiYAKe, ${ }^{1}$ Kanefumi Yamamoto, ${ }^{1}$ Yasujiro MoRIMITSU ${ }^{2}$ and Toshihiko OsaWA ${ }^{2}$ \\ ${ }^{1}$ Central Research Laboratory of Pokka Corporation, Ltd., 45-2 Kumanosyo, Shikatsu-cho, Nishikasugai-gun, Aichi 481, Japan \\ ${ }^{2}$ Department of Applied Biological Sciences, Nagoya University, Nagoya 464-01, Japan
}

Received June 12, 1997; Accepted September 27, 1997

\begin{abstract}
We investigated the antioxidative flavonoid glycosides in the peel extract of lemon fruit (Citrus limon). Six flavanon glycosides: eriocitrin, neoeriocitrin, narirutin, naringin, hesperidin, and neohesperidin, and three flavone glycosides: diosmin, 6,8-di- $C$ - $\beta$-glucosyldiosmin (DGD), and 6- $C$ - $\beta$-glucosyldiosmin (GD) were identified by highperformance liquid chromatography (HPLC) analysis. Their antioxidative activity was examined using a linoleic acid autoxidation system. The antioxidative activity of eriocitrin, neoeriocitrin and DGD was stronger than that of the others. Flavonoid glycosides were present primarily in the peel of lemon fruit. There was only a small difference in the content of the flavonoid glycosides of the lemon fruit juice from various sources and varieties. Lemon fruit contained abundant amounts of eriocitrin and hesperidin and also contained narirutin, diosmin, and DGD, but GD, neoeriocitrin, naringin, and neohesperidin were present only in trace amounts. The content of DGD, GD, and eriocitrin was especially abundant in lemons and limes; however, they were scarcely found in other citrus fruits. The content of flavonoid compounds in lemon juice obtained by an in-line extractor at a juice factory was more abundant than that obtained by hand-squeezing. These compounds were found to be stable even under heat treatment conditions $\left(121^{\circ} \mathrm{C}, 15 \mathrm{~min}\right)$ in acidic solution.
\end{abstract}

Keywords: lemon fruit, Citrus limon, antioxidant, flavonoid glycoside

Lemons (Citrus limon) are used in various food preparations such as soft drinks, alcoholic drinks, and jams, and they are one of the most popular citrus fruits in the world. Lemons, which contain a number of nutrients such as citric acid, ascorbic acid, minerals, and flavonoids, have been known for a long time as typical healthy foods. Flavonoid compounds are widespread in the plant kingdom and comprise a large group of naturally occurring compounds found in all vascular plants. They are present in citrus as well as in other fruits, vegetables, nuts, seeds, grains, tea, and wine. Flavonoid compounds that exhibit beneficial effects on capillary permeability and fragility were once known as vitamin P; they have been investigated regarding their physiological functions, such as hypotensive, antiallergic, anti-inflammatory, antiviral, anticancer, and anticarcinogenic properties (Bracke et al, 1994; Middleton \& Kandaswami, 1994; Mabry \& Ulubelen, 1980).

It has been reported that lemon juice has desmutagenic and antimutagenic effects (Achiwa et al., 1991; Jain et al., 1987) in concern with the prevention of carcinogenesis. Recently, we have been involved in the isolation of antioxidative flavonoid glycosides from lemon fruit and have identified eriocitrin (eriodictyol-7-rutinoside), a flavanone glycoside (Miyake et al, 1997a), and 6,8-di-C- $\beta$-glucosyldiosmin (DGD) and 6- $C$ - $\beta$-glucosyldiosmin (GD), $C$-glucosyl flavones (Miyake et al., 1997b). Lipid peroxidation is known as one of the major factors which causes deterioration of foods during storage and processing. It is also suspected to be strongly associated with aging and carcinogenesis (Yagi, 1987; Cutler, 1984; Harman, 1982). Recently, it was reported that natural antioxidants contained in dietary plants may play an important role in the prevention of carcinogenesis and in extending the life span of animals and may offer effective protection against peroxidative damage in living systems (Jacob, 1995; Cutler, 1992; Osawa et al., 1990). Therefore, much attention has been focused on the importance of natural antioxidants.

In this study, we paid great attention to the antioxidative flavonoid glycosides in lemon fruit. We studied the antioxidative activity and the stability after heat treatment of the flavonoid glycosides to examine their properties for use in foods. We also examined for the distribution of flavonoid glycosides in various sources or varieties of lemon fruit in other citrus fruits.

\section{Materials and Methods}

Materials Lemons (Citrus limon BuRM. f.) and the other citrus fruits were purchased from a supermarket. The varieties of lemon, eureka, lisbon, and verna were obtained from the Citrus Research \& Education Center (Lake Alfred, Florida, USA). The lemon juice from a juice factory was squeezed using an in-line extractor (FMC Co., Lakeland, Florida, USA).

Chemicals Linoleic acid and $\alpha$-tocopherol were obtained from Wako Pure Chemical Industries, Ltd. (Osaka). Flavonoid glycosides were obtained from Funakoshi, Ltd. (Tokyo). Eriocitrin, DGD, and GD were purified according to the method of Miyake et al. (1997a, b.)

Isolation of antioxidative flavonoid glycosides from peel of lemon fruit The peel of lemon fruit $(1.50 \mathrm{~kg})$ was chopped in a homogenizer and extracted with methanol ( $3 l$ ) at room temperature for 3 days. The extract was concentrated 
in vacuo. The concentrated peel extract $(110 \mathrm{~g})$ was chromatographed on a Cosmosil 75C 18-OPN ODS column (Nacalai Tesque, Inc., Kyoto, $\phi 37 \times 500 \mathrm{~mm}$ ). The column was washed with $2 l$ of $20 \%$ methanol-water and was eluted with $40 \%$ methanol-water. The eluate was concentrated in vacuo, and the antioxidative flavonoid fraction $(6.00 \mathrm{~g})$ was obtained. According to the method of Miyake et al. (1997a), the antioxidative flavonoids glycosides were isolated from the antioxidative flavonoid fraction by preparative high-performance liquid chromatography (HPLC). Eriocitrin (1.04 $\mathrm{g})$, neoeriocitrin $(2.30 \mathrm{mg})$, narirutin $(20.5 \mathrm{mg})$, DGD (52.0 $\mathrm{mg}$ ), and GD (15.8 $\mathrm{mg}$ ) were isolated. Eriocitrin, DGD, and DG were identified by MS, ${ }^{1} \mathrm{H}-\mathrm{NMR}$, and ${ }^{13} \mathrm{C}-\mathrm{NMR}$ analyses (Miyake et al, 1997a, b). Neoeriocitrin and narirutin were identified from the retention time of standard reagents for HPLC analysis. The aglycones of neoeriocitrin and narirutin were prepared by hydrolysis using pectinase according to the method of Miyake et al. (1997a). Also, it was confirmed that the retention time of these aglycones was compatible with that of the standard reagent, eriodictyol and naringenin, during HPLC analysis. Other flavonoid glycosides of naringin, hesperidin, neohesperidin, and diosmin were identified from the retention time of standard reagents for HPLC analysis of the antioxidative flavonoid fraction (Fig. 1). HPLC (LC10A, Shimadzu Co., Ltd., Kyoto) was carried out using a Shim-pack CLC-ODS(M) $(4.6 \times 250 \mathrm{~mm})$ with a UV detector $(\lambda=280 \mathrm{~nm})$. The solvent system contained $70 \%$ of a $5 \%$ acetic acid solution and $30 \%$ methanol. The flow rate was $1.0 \mathrm{ml} /$ $\min$.

Antioxidative assay Antioxidative activity was evaluated using the linoleic acid system (Osawa \& Namiki, 1981). Each sample was added to a mixed solution of linoleic acid $(0.13 \mathrm{ml})$ and $99.0 \%$ distilled ethanol $(10 \mathrm{ml})$ with a $50 \mathrm{~mm}$ phosphate buffer $(\mathrm{pH} 7.0,10 \mathrm{ml})$, and the volume was adjusted to $25 \mathrm{ml}$ with distilled water. The reaction mixture in a conical flask was incubated at $40^{\circ} \mathrm{C}$. At intervals during the incubation, the degree of oxidation was measured using the thiocyanate method (Mitsuda et al., 1966) by recording the absorbance at $500 \mathrm{~nm}$ after coloring with $\mathrm{FeCl}_{2}$ and thiocyanate. $\alpha$-Tocopherol was used as the positive standard.

Determination of flavonoid glycosides The lemon fruit, from a Californian source, was separated into the peel, juice, and seed in order to determine the location of eriocitrin. The peel was further separated into the flavedo (epicarp), albedo (mesocarp), and pulp vesicles using a knife. Samples $(5.0 \mathrm{~g})$ of the subdivided peel and the seed were homogenized and extracted with $100 \mathrm{ml}$ of methanol at $37^{\circ} \mathrm{C}$ for 4 days. The pulp of the squeezed juice was removed by filtration.

Each citrus fruit was separated into the peel and juice by hand-squeezing the fruit. The peel $(5.0 \mathrm{~g})$ was homogenized and extracted with $100 \mathrm{ml}$ of methanol at $37^{\circ} \mathrm{C}$ for 4 days. The pulp of the squeezed juice was removed by filtration. Flavonoid glycosides in the extract of the peel and in the filtered juice were determined by HPLC analysis according to the previous method.

Stability of flavonoid glycosides by heat treatment at different $\mathrm{pH}$ The buffer solutions were at $\mathrm{pH} 2$ and $\mathrm{pH} 3.5$ using a $100 \mathrm{mM}$ sodium citrate- $\mathrm{HCl}$ buffer, and at $\mathrm{pH} 5.0$ and pH 7.0 using a $100 \mathrm{mM}$ sodium phosphate buffer. The final

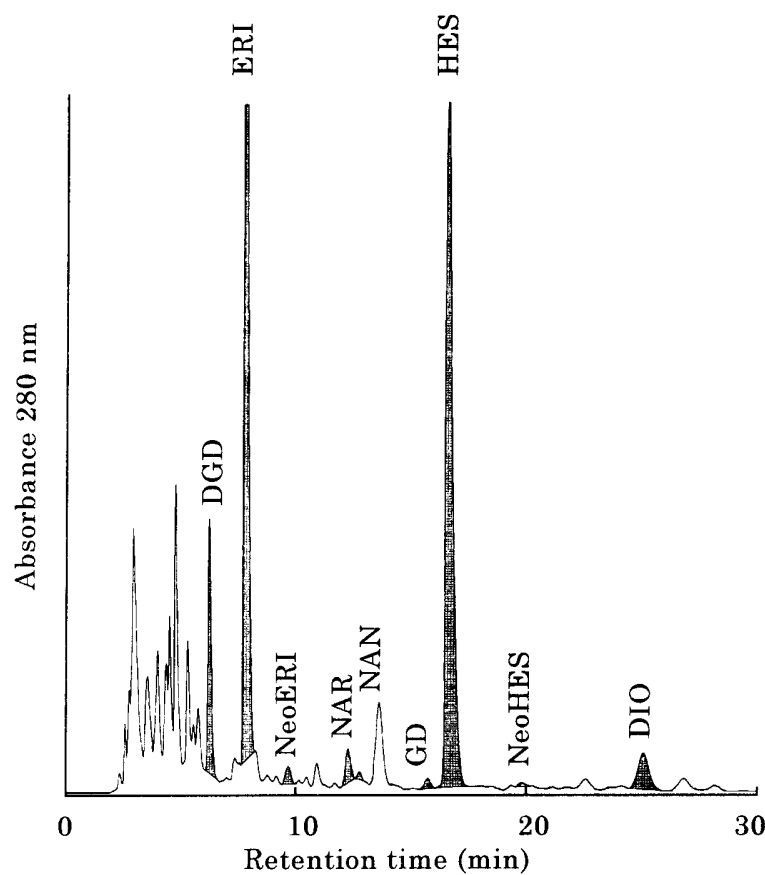

Fig. 1. HPLC profile for the antioxidative flavonoid fraction. ERI: eriocitrin, NeoERI: neoeriocitrin, NAR: narirutin, NAN: naringin, HES: hesperidin, NeoHES: neohesperidin, DIO: diosmin, DGD: 6,8 -di- $C$ - $\beta$-glucosyldiosmin, GD: $6-C$ - $\beta$-glucosyldiosmin

concentration of $0.1 \%$ flavonoid glycosides was added to the different buffer solutions, and the solutions were heated at $80^{\circ} \mathrm{C}$ for $30 \mathrm{~min}$ or at $121^{\circ} \mathrm{C}$ for $15 \mathrm{~min}$. The stability of the flavonoid glycosides after heat treatment was determined by the residual ratio of the flavonoid glycosides after heat treatment. The residual ratio of eriocitrin was determined from the residual peak of eriocitrin after heat treatment by HPLC analysis.

\section{Results and Discussion}

Antioxidative flavonoid compounds in lemon fruit We previously reported that flavonoid fractions prepared from peel extract or juice using ODS column chromatography exhibited strong antioxidative activity in the linoleic acid autooxidation system (Miyake et al., 1997a). The antioxidants were identified as eriocitrin (eriodictyol $\beta$-7-rutinoside), a flavanone glycoside, and as DGD and GD, C-glucosyl flavones (Miyake et al., 1997b). We further isolated another two antioxidative flavonoid glycosides from peel extract. They were identified as neoeriocitrin (eriodictyol $\beta$-7-rhamnoglucoside) and narirutin (naringenin $\beta$-7-rutinoside) Figure 1 shows the HPLC profile of the antioxidative flavonoid fraction. The other flavonoid compounds were identified as naringin (naringenin $\beta$-7-rhamnoglucoside), hesperidin (hesperetin $\beta$-7-rutinoside), and diosmin (diosmetin $\beta$-7-rutinoside) from the antioxidative flavonoid fraction. We identified nine flavonoid glycosides in the antioxidative flavonoid fraction in the peel extract of the lemon fruit, and their structures are shown in Fig. 2.

The antioxidative activity of the nine flavonoid glycosides was examined using the linoleic acid autoxidation system as 
shown in Fig. 3. We previously reported the antioxidative activity of eriocitrin, DGD and GD in the linoleic acid system (Miyake et al, 1996a, b). Eriocitrin had the strongest antioxidative activity among the flavonoid glycosides in lemon fruit. Neoeriocitrin and DGD also showed antioxidative activity, but the others had only a weak activity. We expect that the antioxidants in lemon fruit will provide protection against peroxidative damage in living systems in relation to aging and carcinogenesis.
Properties of flavonoid compounds The properties of the flavonoid compounds in lemon fruit were examined for solubility in water or ethanol. These compounds were soluble in ethanol, but GD, hesperidin, neohesperidin, and diosmin were insoluble in water. The taste of these flavonoid glycosides $(0.1 \%$ water solution) was examined. Naringin, neoeriocitrin, and neohesperidin having the sugar chain of neohesperidose (2- $O$ - $\alpha$-L-rhamnosyl- $\beta$-D-glucose) were more bitter than narirutin, eriocitrin, and hesperidin having the sugar

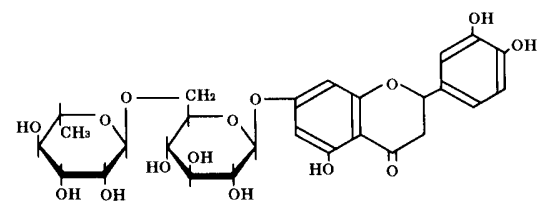

eriocitrin (ERI)

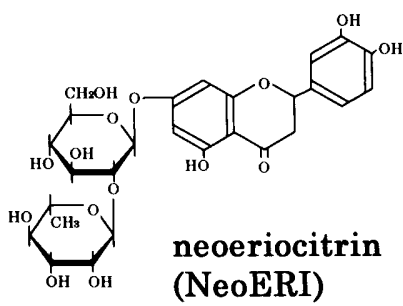

(NeoERI)

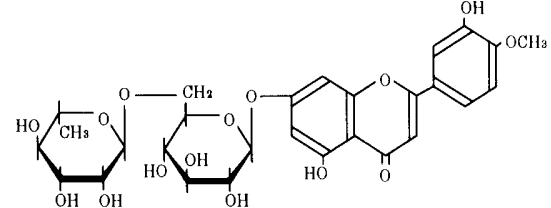

diosmin (DIO)

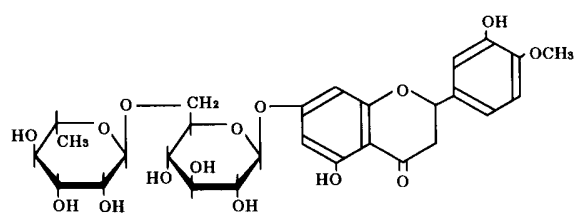

hesperidin (HES)

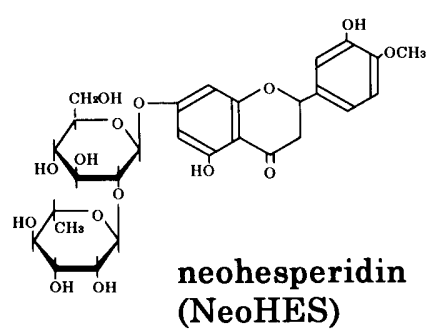

(NeoHES)

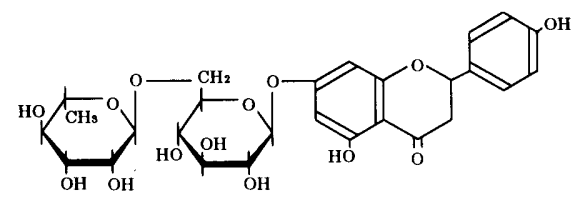

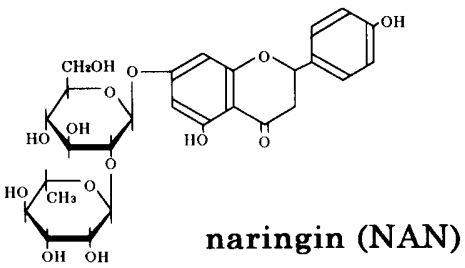

6,8-di- $C$-glucosyldiosmin (DGD)

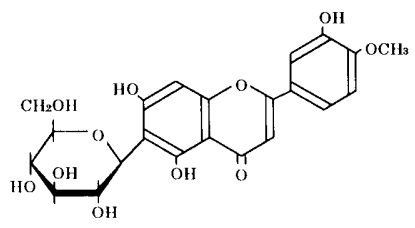

narirutin (NAR)

Fig. 2. Structure of flavonoid compounds in lemon fruit.

6- $C$-glucosyldiosmin (GD)

Lipid peroxidation (\%)

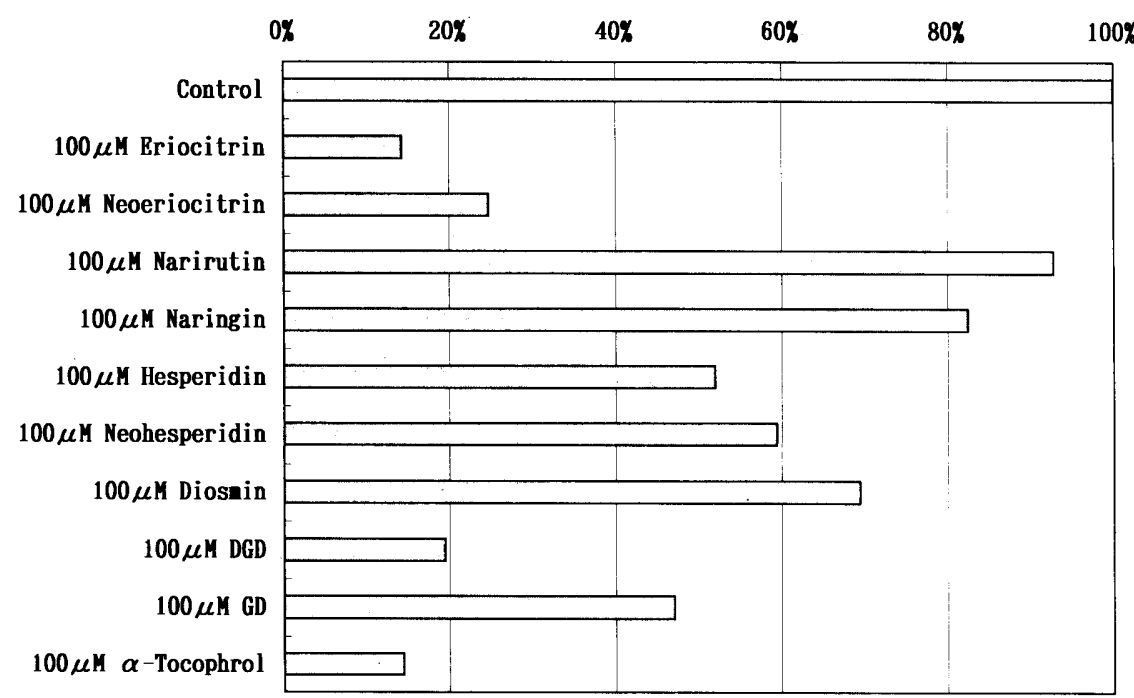

Fig. 3. Antioxidative activity of flavonoid glycosides. DGD : 6,8-di- $C$ - $\beta$-glucosyldiosmin, GD : 6- $C$ - $\beta$-glucosyldiosmin. 
chain of rutinoside (6-O- $\alpha$-L-rhamnosyl- $\beta$-D-glucose). These findings suggest that the bitterness of citrus juice is related to the structure of the neohesperidose portion of the flavonoid glycosides.

We examined the stability of flavonoid glycosides under conditions of sterilization because of the importance of this process in the manufacture of beverages and foods. The stability of flavonoid compounds after heat treatment in different buffer solutions of $\mathrm{pH} 2.0, \mathrm{pH} 3.5, \mathrm{pH} 5.0$, and $\mathrm{pH}$ 7.0 was also examined. The heat treatment at $80^{\circ} \mathrm{C}$ for $30 \mathrm{~min}$ assuming the sterilization of yeast or mold was used, and the heat treatment at $121^{\circ} \mathrm{C}$ for $15 \mathrm{~min}$ assuming the sterilization of heat-stable bacteria was used. For all of the flavonoid glycosides, except eriocitrin and neoeriocitrin, more than $90 \%$ remained after heat treatment at $80^{\circ} \mathrm{C}$ for $30 \mathrm{~min}$, and more than $80 \%$ remained after heat treatment at $121^{\circ} \mathrm{C}$ for $15 \mathrm{~min}$. These compounds were fairly stable under the heat treatment conditions. Eriocitrin and neoeriocitrin were unstable in a neutral solution $\left(\mathrm{pH} \mathrm{7.0)}\right.$ at $80^{\circ} \mathrm{C}$ for $30 \mathrm{~min}$ and $121^{\circ} \mathrm{C}$ for 15 min, these compounds were decreased to about half of the initial content after heating at $80^{\circ} \mathrm{C}$ for $30 \mathrm{~min}$ and almost completely disappeared after heat treatment at $121^{\circ} \mathrm{C}$ for 15 min. It is suggested that these compounds having strong antioxidative activity changed by oxidation under the previous conditions, as well as ascorbic acid, which is an antiox- idant and unstable under high temperature in neutral solution. The chemical and physical properties of flavonoid compounds in citrus fruit have been reported (Rouseffy, 1980). It has been reported that the A and B rings in the flavonoid molecule are stable and relatively unreactive, and that the central $\mathrm{C}$ ring of the central $\mathrm{C}_{3}$ group is the most reactive portion of the molecule and undergoes a ring opening reaction with a base or acetic anhydride (Hatori, 1962). The decrease in eriocitrin and neoeriocitrin by heat treatment in a neutral solution may be due to such ring opening reaction. The $\mathrm{C}$ ring of these compounds having the catecol structure of the adjacent dihydroxy groups on the B-ring seemed to be opened easier than that of other flavonoid compounds. These results suggest that the antioxidative flavonoid compounds such as eriocitrin and neoeriocitrin, which are stable after heat treatment in the acidic condition of $\mathrm{pH}$ 3.5-5.0, may be suitable for use in acidic beverages and foods.

Flavonoid compounds in citrus fruit have generally been reported to be stable under storage conditions (Ranganna et al., 1983). In commercial lemon juice, the nine flavonoid compounds were stable during sterilization at $80^{\circ} \mathrm{C}$ for $30 \mathrm{~min}$ and under storage conditions (room temperature for 9 months), and the content of ascorbic acid decreased to $60 \%$ under the same conditions (data not shown.) The stability of

Table 1. Distribution of flavonoid compounds in each variety and source of lemon juice.

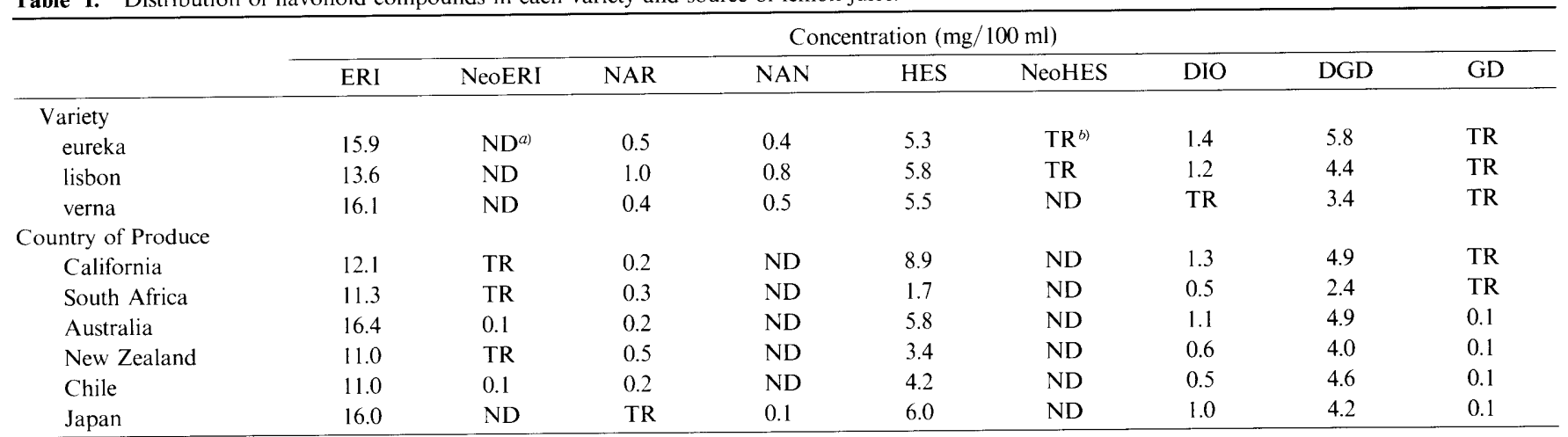

a) not detected.

${ }^{b}$ trace: less than $0.1 \mathrm{mg} / 100 \mathrm{ml}$

ERI: eriocitrin, NeoERI: neoeriocitrin, NAR: narirutin, NAN: naringin, HES: hesperidin, NeoHES: neohesperidin, DIO: diosmin, DGD: $6,8-\mathrm{di}-C-\beta$-glucosyldiosmin, GD: $6-C-\beta$-glucosyldiosmin.

Table 2. Distribution of flavonoid compounds in various locations in lemon fruit.

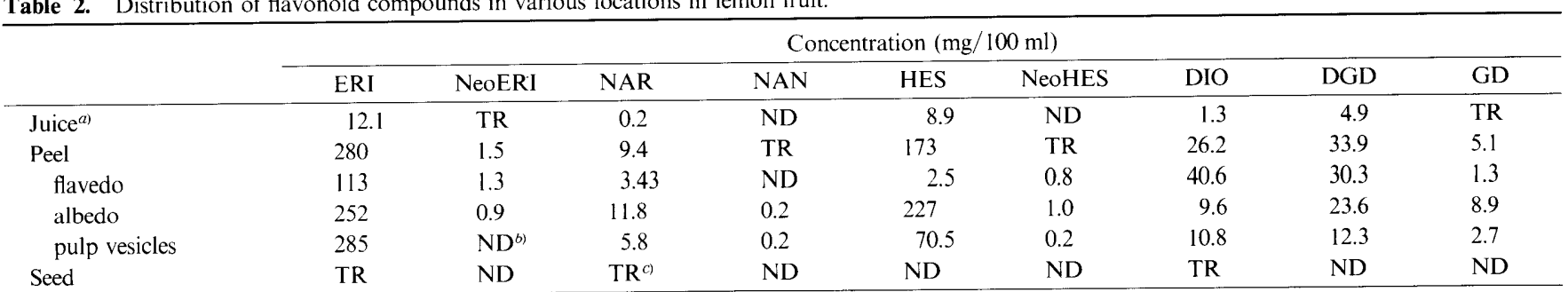

a) The content of juice represented with flavonoid compounds $(\mathrm{mg})$ of $100 \mathrm{ml}$ juice $(\mathrm{mg} / 100 \mathrm{ml})$.

${ }^{b)}$ not detected.

c)trace: less than $0.1 \mathrm{mg} / 100 \mathrm{~g}$.

ERI: eriocitrin, NeoERI: neoeriocitrin, NAR: narirutin, NAN: naringin, HES: hesperidin, NeoHES: neohesperidin, DIO: diosmin, DGD: 6,8 -di- $C$ - $\beta$-glucosyldiosmin, GD: 6-C- $\beta$-glucosyldiosmin. 
flavonoid compounds under storage conditions of the lemon juice was greater than that of ascorbic acid.

Determination of flavonoid glycosides in lemon fruit As shown in Table 1, the content of flavonoid glycosides in the juice of different varieties of lemon fruit (eureka, lisbon, and verna) and in the juice of fruit produced in different countries (California, South Africa, Australia, New Zealand, Chile, and Japan) was examined. It was found that the content of flavonoid compounds was fairly similar among the varieties and the sources of the lemon fruit.

The content of flavonoid compounds in various parts of the lemon fruit was examined (Table 2). All of the nine flavonoid compounds were found to be more abundant in the peel than in the juice, but only a small amount of these compounds was found in the seed. Eriocitrin and hesperidin existed abundantly in the peel and juice. The content of

Table 3. Content of flavonoid compounds in lemon juice obtained by hand squeezing and factory squeezing.

\begin{tabular}{|c|c|c|c|c|c|c|c|c|c|c|c|c|}
\hline & \multicolumn{12}{|c|}{ Concentration $(\mathrm{mg} / 100 \mathrm{ml})$} \\
\hline & ERI & NeoERI & NAR & NAN & HES & NeoHES & $\mathrm{DIO}$ & DGD & GD & Brix & $\mathrm{pH}$ & Acidity \\
\hline Hand squeezing & 12.5 & 0.3 & 0.3 & $\mathrm{ND}^{a)}$ & 8.9 & ND & 2.1 & 7.2 & ND & 8.5 & 2.5 & 4.8 \\
\hline In-line extractor & 19.8 & 0.3 & 0.8 & 0.3 & 12.2 & 0.2 & 3.4 & 9.7 & 0.2 & 8.9 & 2.4 & 5.5 \\
\hline
\end{tabular}

a) not detected.

ERI: eriocitrin, NeoERI: neoeriocitrin, NAR: narirutin, NAN: naringin, HES: hesperidin, NeoHES: neohesperidin, DIO: diosmin, DGD: 6,8 -di- $C$ - $\beta$-glucosyldiosmin, GD: 6-C- $\beta$-glucosyldiosmin.

Table 4. Distribution of flavonoid compounds in citrus juices.

\begin{tabular}{|c|c|c|c|c|c|c|c|c|c|}
\hline & \multicolumn{9}{|c|}{ Concentration $(\mathrm{mg} / 100 \mathrm{ml})$} \\
\hline & ERI & NeoERI & NAR & NAN & HES & NeoHES & $\mathrm{DIO}$ & DGD & GD \\
\hline Citrus. limon (lemon) & 12.1 & $\mathrm{TR}^{a)}$ & 0.2 & $\mathrm{ND}^{b)}$ & 8.9 & ND & 1.3 & 4.9 & TR \\
\hline C. aurantifolia (lime) & 9.7 & TR & 0.6 & ND & 10.2 & ND & 0.3 & 2.2 & 0.1 \\
\hline C. sinensis (valencia orange) & 0.2 & TR & 22.9 & ND & 32.1 & ND & ND & ND & ND \\
\hline C. sinensis (navel orange) & 0.3 & 0.2 & 14.8 & 0.3 & 37.4 & ND & $\mathrm{ND}$ & ND & ND \\
\hline C. unshiu (unshiu) & 0.3 & TR & 26.4 & TR & 30.1 & ND & TR & ND & ND \\
\hline C. iyo (iyo) & ND & ND & 10.0 & ND & 12.3 & ND & ND & ND & ND \\
\hline C. reticulata (ponkan) & 0.1 & ND & 3.6 & ND & 15.3 & ND & ND & ND & ND \\
\hline C. paradisi (grapefruit, white) & 0.1 & ND & 18.6 & 390 & 0.9 & 2.9 & ND & ND & ND \\
\hline C. paradisi (grapefruit, pink) & 0.1 & 0.2 & 8.5 & 27.0 & 0.5 & 1.5 & ND & ND & ND \\
\hline C. aurantium (daidai) & ND & 8.4 & 0.6 & 22.3 & ND & 12.9 & ND & ND & ND \\
\hline C. hassaku (hassaku) & 0.1 & 0.3 & 8.6 & 28.5 & 1.8 & 9.7 & ND & ND & ND \\
\hline C. sphaerocarpa (kabosu) & ND & ND & 16.4 & 1.8 & 2.8 & 1.1 & ND & TR & ND \\
\hline C. junos (yuzu) & 0.1 & 0.1 & 15.0 & 5.4 & 6.5 & 3.3 & ND & ND & ND \\
\hline
\end{tabular}

a) trace: less than $0.1 \mathrm{mg} / 100 \mathrm{ml}$.

${ }^{b)}$ not detected.

ERI: eriocitrin, NeoERI: neoeriocitrin, NAR: narirutin, NAN: naringin, HES: hesperidin, NeoHES: neohesperidin, DIO: diosmin, DGD: 6,8 -di- $C$ - $\beta$-glucosyldiosmin, GD: 6-C- $\beta$-glucosyldiosmin.

Table 5. Distribution of flavonoid compounds in each variety and source of lemon juice.

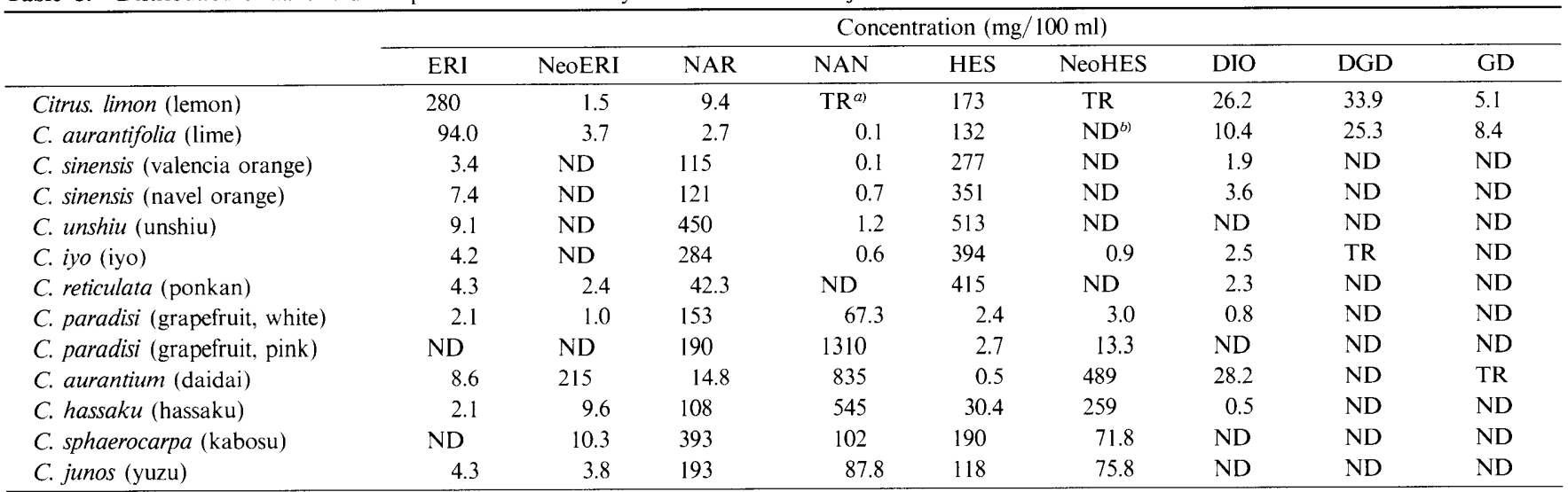

a) trace: less than $0.1 \mathrm{mg} / 100 \mathrm{~g}$.

${ }^{b)}$ not detected.

ERI: eriocitrin, NeoERI: neoeriocitrin, NAR: narirutin, NAN: naringin, HES: hesperidin, NeoHES: neohesperidin, DIO: diosmin, DGD: 6 ,8-di- $C$ - $\beta$-glucosyldiosmin, GD: 6-C- $\beta$-glucosyldiosmin. 
eriocitrin in the albedo (mesocarp, white interior of the peel) and pulp vesicles (in part of the enveloping flesh) was more abundant than in the flavedo (epicarp, yellow exterior of the peel), and the hesperidin content was found to be abundant in the albedo. DGD, narirutin, and diosmin existed in various parts of the peel and juice, but only small amounts of neoeriocitrin, naringin, GD, and neohesperidin were detected.

The concentration in the juice of the lemon fruit of ascorbic acid, which is used as an antioxidant in food products and has biofunctional activity in vivo (Jacob, 1995), was about $45 \mathrm{mg}$ in $100 \mathrm{ml}$ of juice. Ascorbic acid is an important antioxidant in the juice of the lemon fruit but was unstable under the food processing conditions of heat treatment and food sterilization, and under food storage conditions. The total concentration of the above nine flavonoid compounds, which are stable under conditions of sterilization of foods and beverages, was 37.4 $\mathrm{mg}$ in $100 \mathrm{ml}$ of juice and that of eriocitrin, the strong antioxidative flavonoid compound, it was $12.1 \mathrm{mg}$ in $100 \mathrm{ml}$ of juice. The flavonoid compounds seemed to be important antioxidants, as well as ascorbic acid, because they exist in a considerable amount in the juice of the lemon fruit and are stable under normal processing or storage conditions.

The content of flavonoid compounds in lemon juice was examined relative to the method for squeezing the fruit by an in-line extractor or by hand squeezing (as shown in Table 3). The content of eriocitrin and hesperidin in lemon juice obtained by an in-line extractor was greater than that of the juice obtained by hand squeezing. We estimated that the juice obtained by an in-line extractor contained abundant amounts of eriocitrin and hesperidin because pulps, which are parts of the albedo and pulp vesicle in peel, were contaminated in the juice. The in-line extractor method of squeezing fruit is important commercially for the better yield of the juice. Using this method, the lemon fruit was squeezed with higher pressure than by hand squeezing. Because of the pressure on the fruit, parts of the peel, the albedo and pulp vesicle, in which eriocitrin and hesperidin are abundant, were present in the juice as pulp.

Distribution of flavonoid compounds in citrus fruits The distribution of flavonoid compounds from the peel (Table 4) and juice (Table 5) of citrus fruits was investigated. Eriocitrin, DGD and GD were also shown to exist in limes as well as lemons, but very little was present in the other types of citrus fruits. Little neoeriocitrin was found in lemons, but it was found to be especially rich in daidai. Eriocitrin and GD were also shown to exist in limes as well as in lemons, but very little was present in the other types of citrus fruits. These compounds were found to be especially plentiful in lemon and lime fruits and abundant in the peel obtained as a byproduct from juice factories. These results suggested that effective utilization of the peel in juice products will make available a greater amount of antioxidative flavonoid compounds.

\section{References}

Achiwa, Y., Koda, T. and Namiki, K. (1991). Inhibition of N-nitrosomorpholine formation by lemon juice. Nippon Shokuhin Kogyo Gakkaishi, 38, 826-830 (in Japanese).

Bracke, M.E., Bruyneel, E.A., Vermeulen, S.J., Vennekens, K., Marck, V.V. and Mareel, M.M. (1994). Citrus flavonoid effect on tumor invasion and metastasis. Food Technol, 48, 121-124.

Cutler, R.G. (1984). Antioxidants, aging, and longevity. In "Free Radicals in Biology," ed. by W.A. Pryor. Academic Press, Orlando FL, Vol. 6, pp. 371-423.

Cutler, R.G. (1992). Genetic stability and oxidative stress: common mechanism in aging and cancer. In "Free Radicals and Aging," ed. by I. Emerit and B. Chance. Birkhauser Verlag, Basel, Switzerland, pp. $31-46$.

Harman, D. (1982). The free-radical theory of aging. In "Free Radicals in Biology," ed. by W.A. Pryor. Academic Press, Orlando, FL, Vol. 5, Chapter 8.

Hatori, S. (1962). In "The Chemistry of Flavonoid Compounds," ed by T. Geissman. MacMillan, New York, pp. 286.

Jacob, R.A. (1995). The integrated antioxidant system. Nutr. Res., 15, 755-766.

Jain, A.K., Shimoi, K., Nakamura, Y., Tomita, I. and Koda, T. (1987). Preliminary study on the desmutagenic and antimutagenic effect of some natural products. Curr. Sci., 56, 1266-1269.

Mabry, T.J. and Ulubelen, A. (1980). Chemistry and utilization of phenypropanoids including flavonoids, coumarins and lignans. $J$. Agric. Food Chem., 28, 188-196.

Middleton, E. and Kandaswami, C. (1994). Potential health-promoting properties of citrus flavonoids. Food Technol, 48, 115-119.

Mitsuda, H., Yamamoto, K. and Iwami, K. (1966). Antioxidative action of indole compounds during the autooxidation of linoleic acid. Eiyo to Shokuryo, 19, 210-214 (in Japanese).

Miyake, Y., Yamamoto, K. and Osawa, T. (1997a). Isolation of eriocitrin (eriodictyol 7-rutinoside) from lemon fruit (Citrus limon Burm. f.) and its antioxidative activity. Food Sci. Technol. Int. Tokyo, 3, 84-89.

Miyake, Y., Yamamoto, K., Morimitsu, Y. and Osawa, T. (1997b). Isolation of $C$-glucosylflavone from lemon peel and antioxidative activity of flavonoid compounds in lemon fruit. J. Agric. Food Chem., 45, 4619-4623.

Osawa, T. and Namiki, M. (1981). A novel type of antioxidant isolated from leaf wax of Eucalyptus leaves. Agric. Biol. Chem., 45, 735-739.

Osawa, T., Namiki, M. and Kawakishi, S. (1990). Role of dietary antioxidants in protection against oxidative damage. In "Antimutagenesis and Anticarcinogenesis Mechanisms," ed. by Y. Kuroda, D.M. Shankel and M.D. Waters. Plenum Publishing, New York, pp. 139-153.

Ranganna, S., Govindarajan, V.S. and Ramana, K.V.R. (1983). Citrus fruits - varieties, chemistry, technology, and quality evaluation. Part II. Chemistry, technology, and quality evaluation. A. Chemistry. Crit. Rev. Food Sci. Nutr. P, 18, 313-386.

Rouseff, R.L. (1980). Flavonoid and citrus quality. In "Citrus Nutrition and Quality," ed. by S. Nagy and J.A. Attaway. American Chemical Society Publishing, Washington, D.C., pp. 83-108.

Yagi, K. (1987). Lipid peroxides and human disease. Chem. Phys. Lipids, 45, 337-341. 